\title{
Exploring Web Partition in DHT-Based Distributed Web Crawling
}

\author{
$\mathrm{Xiao}_{\mathrm{XU}}^{\dagger \mathrm{a})}$, Nonmember, Weizhe $\mathrm{ZHANG}^{\dagger}$, Member, Hongli ZHANG ${ }^{\dagger}$, and Binxing FANG $^{\dagger}$, Nonmembers
}

\begin{abstract}
SUMMARY The basic requirements of the distributed Web crawling systems are: short download time, low communication overhead and balanced load which largely depends on the systems' Web partition strategies. In this paper, we propose a DHT-based distributed Web crawling system and several DHT-based Web partition methods. First, a new system model based on a DHT method called the Content Addressable Network (CAN) is proposed. Second, based on this model, a network-distance-based Web partition is implemented to reduce the crawler-crawlee network distance in a fully distributed manner. Third, by utilizing the locality on the link space, we propose the concept of link-based Web partition to reduce the communication overhead of the system. This method not only reduces the number of inter-links to be exchanged among the crawlers but also reduces the cost of routing on the DHT overlay. In order to combine the benefits of the above two Web partition methods, we then propose 2 distributed multi-objective Web partition methods. Finally, all the methods we propose in this paper are compared with existing system models in the simulated experiments under different datasets and different system scales. In most cases, the new methods show their superiority.

key words: DHT, distributed Web crawling, Web partition, content addressable network, communication overhead
\end{abstract}

\section{Introduction}

Web search services are becoming more and more important in everyone's daily life. Their availability and effectiveness largely depends on the efficiency of the underlying crawling systems. Nowadays, due to the huge evolution of the Web in the last 10 years, building a practical and effective search engine system has become an extremely complicated task involving both intelligence and funds which keeps smaller companies out of the door. Inspired by the concept of internet computing [1] and SETI@ home [2], large scale distributed Web crawling (DWC) systems using idle computing resources e.g. idle servers in IDCs and home computers across the Internet has been applied in practice. Catering to this trend, DHT-based distributed Web crawling is proposed to handle task scheduling and node churn in a fully distributed manner providing a new system model with enhanced robustness and scalability.

In this paper, we propose our own DHT-based DWC system and several Web partition methods based on the system. Our goal can be described as follows: Given a set of crawlers and a set of Web hosts distributed across the internet, we want to establish the crawler-host relationship in which each crawler is responsible for crawling a set of Web

\footnotetext{
Manuscript received February 25, 2010.

Manuscript revised May 10, 2010.

${ }^{\dagger}$ The authors are with Harbin Institute of Technology, China.

a) E-mail: smilestor@gmail.com

DOI: 10.1587/transinf.E93.D.2907
}

hosts. The crawler-host relationship is achieved so that the Web content can be pulled into the system as fast as possible. At the same time, the communication overhead generated by the crawlers is small. The problem should be solved in a distributed manner to avoid single point of failure in the dynamic internet environment.

In order to reduce the download time cost of each Web page, a Web host should be crawled by the crawlers located nearest to it on the Internet. We call the distance between a crawler and a Web host as the network distance. In our system, this value equals to the combination of the average round trip time (RTT) and average download time (DLT) between the crawler-host pair. In order to keep the network distance short, we propose a new system model based on the network coordinate system (NC). First, each Web host and crawler is assigned a unique network coordinate calculated according to the network distances between them by the NC. Then, a DHT is built on the coordinate space. The crawlers join the DHT using their coordinates as IDs; while the Web hosts are inserted into the DHT using their coordinates as keys. Through this process, the crawlers divide the DHT's key space (the network coordinate space) in a fully distributed manner and establish a network-distance-based Web partition under which each Web host is assigned to a crawler located close to it in the NC space.

On the other hand, in order to maintain high coverage and balance the work load, DWC systems usually adopt URL exchange method. In these systems, the distributed crawlers exchange newly discovered inter-links [3]. The communication overhead generated by this kind of exchanging occupy the bandwidth, the memory space and CPU slices of the heavily loaded crawling servers. In this paper, we propose a new method to significantly reduce the number of inter-links to be exchanged among the crawlers thus reducing the overall communication overhead. In our system, the Web hosts are assigned keys calculated according to the topology of the links. A DHT is built on the key space in order to divide the Web hosts into subsets in a distributed manner and assign the subsets to the crawlers. Under this so called link-based Web partition, if there is high connectivity between a group of Web hosts, the Web hosts are assigned to the same subset (crawler) so that the links between the Web hosts can be processed in the crawler's local memory avoiding inter-crawler communications.

Since our goal is to reduce both the network latencies and the communication overhead, we need a multiobjective Web partition method to achieve the two goals at 
the same time. In this paper, we propose two distributed multi-objective Web partition methods combining the above two partition methods. As both the network position and the link position can be parameterized as the keys of the DHT, such combination can be implemented by either using multiple dimensions in one single key space or using multiple key spaces. The methods are compared with the existing DHT-based DWC system and showed their superiority.

The rest of the paper is organized as follows: Section 2 outlines the related works. Section 3 describes our DHT-based system model. Based on this model, Sect. 4 proposes the network-distance-based Web partition method. Section 5 proposes the link-based Web partition method. By combining the methods proposed in Sect. 4 and 5, Sect. 6 proposes two distributed multi-objective Web partition methods. In Sect. 7, the methods proposed are evaluated through simulations. Finally, in Sect. 8 we conclude the paper and outline the research directions in the future.

\section{Related Works}

The existing DWC systems can be classified into two types: the cluster-based systems and the Internet-based systems. The cluster-based systems such as Mercator [4], the early form of Google [5] have their machines located in the same location both in the network and the physical world. There's no way to reduce the download time since all crawlers subject to the same network distance to any Web host. Moreover, all the URLs (including inter-links) discovered are centrally processed (including URL submission, URL recrawl and removing the seen URLs).

On the other hand, the Internet-based systems have their machines located all over the Internet, so that they can make optimizations in reducing the network distances. However, there's no common agreement on how the network distance should be measured and defined.

Cho, J. [3] first proposed a series of basic concepts on parallel crawling and distributed crawling, including classification methods, evaluation metrics and so on. After 2003, several systems were proposed: UbiCrawler [6] is the first crawling system announced to be deployed on WAN. The consistent hashing [7] method it adopts guarantees the load balancing among crawlers. However, consistent hashing prevents the system from optimizations on network distance. In IPMicra [8], [9], crawler is selected to crawl a certain Web site if they were located within the same AS or ISP network according to the information provided by Regional Internet Registries (RIRs); SE4SEE [10] reduces the network distance by assigning crawler Web sites that were located within the crawler's country. The metrics of network distance the above two systems adopt (AS differences and geographical distances) cannot fully reveal the Web hosts' true positions on the Internet, because the routers' routing strategies usually don't comply with the restrictions of ASs or cities. Apoidea [11] implements a Chord-based DWC system. However, it didn't make optimizations in reducing the network distance.
We have made a contribution on reducing the network distance in [12]. In the works, we first put forward the idea of combining DHT and $\mathrm{NC}$ to reduce the network distance within each crawler-crawlee pair. However, the model of network distance was only derived from the RTTs, which is not exhaustive since the bandwidth also affects the download process. Moreover, the communication overhead was not taken into consideration in that paper.

In order to maintain high coverage and balance the work load, DWC systems such as Apoidea [11], IglooG [13] and Ubicrawler [6] etc. adopt URL exchange method. In these systems, the distributed crawlers exchange newly discovered inter-links which causes a large number of intercrawler communications. To reduce the amount of communications, there are existing methods proposed:

1) Batch exchange [3]: Each crawler packs a number of inter-links in one communication in order to reduce the number of communications.

2) Reducing duplicated links: Each crawler caches its recently discovered links. When a link is discovered, the cache checks whether it is a duplicate. Only the links that don't exist in the cache are to be exchanged.

3) Popular hosts overlapping [3]: The system has a global list of popular Web hosts. Upon the discovery of links pointed to the popular hosts, the crawler simply crawls it. Since the links of the popular hosts are not exchanged among crawlers, the communications can be reduced.

However, the above methods are not the fundamental solutions to the problem as they either cannot reduce the number of unique links to be exchanged or causes overlapping problems.

Many Internet-based DWC systems are built by commercial companies and open source community, but few detailed information was exposed about these systems. So far as we know, Yacy [14] is a distributed search engine providing user-customized search services; Majestic [15] implemented a crawling system using the architecture of SETI@Home [2]; Faroo [16] implemented a fully distributed P2P search engine.

\section{Overview}

\subsection{System Model}

As shown in Fig. 1, our system consists of a number of crawlers and a central module. The main task of the central module is to measure and calculate the IDs and keys of the crawlers and the target Web hosts. The crawlers all join a DHT using the IDs, while the Web hosts are inserted to the same DHT using the keys. The crawlers are responsible for crawling the target Web hosts and exchanging the interlinks among each other. Each crawler only communicates with the central module on rare occasions such as joining and searching for the key of a Web host. In order to keep the retrieved Web data up to date, all the Web hosts will be 


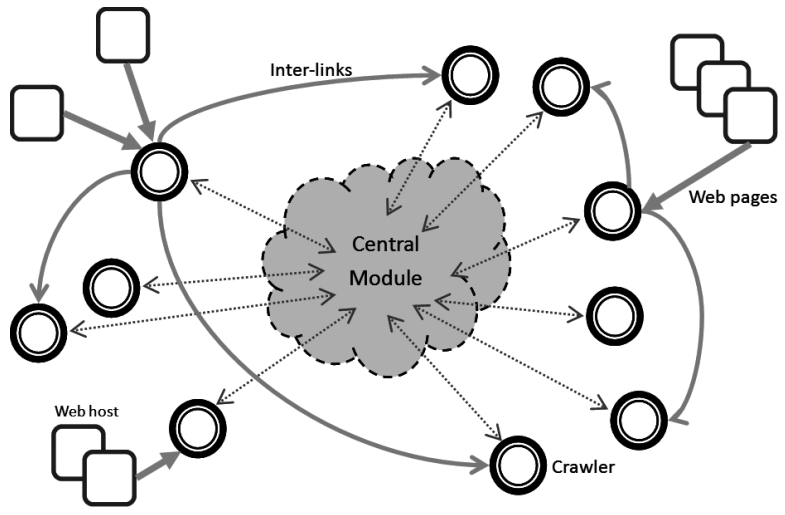

Fig. 1 The diagram of the proposed DWC system.

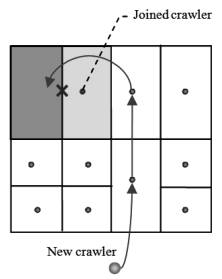

(a) crawler joining

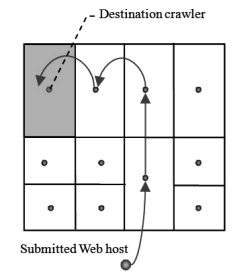

(b) inserting a Web host

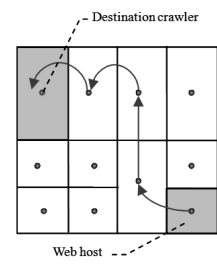

(c) routing an inter-link
Fig. 2 The DHT-based functionalities of the proposed DWC system.

periodically re-crawled.

We use the Content Addressable Network (CAN) as the system's DHT method. As a result, all IDs and keys are multi-dimensional coordinates. According to CAN, the whole coordinate space is dynamically partitioned so that each peer is assigned a distinct space called a zone. A peer holds the information of the other peers whose zones are close to its own. The information is used as routing table, in order to route messages between arbitrary peers in the coordinate space. Upon the joining of each new peer (Fig. 2 (a)), firstly the new peer's coordinate is calculated by the central module and assigned to the new peer as its ID. Then, an already-joined peer's space (or zone) is split into two if the new peer's coordinate falls into that peer's zone. The split is done by assuming a certain ordering of the dimensions in deciding along which dimension a zone is to be split. This splitting method proposed by [17] is effective on balanced space partition and zone re-merging. The new peer picks one of the newly partitioned spaces as its own zone and the already-joined peer continues to hold the remainder space as its zone. Each peer constantly checks the availability of all its direct neighbors in order to discover the un-notified peer departures. The peer departure algorithm is achieved by merging the departed peer's zone with the other living peer's zone using the method proposed in Appendix A of [17].

On the other hand, Web hosts (Fig. 2(b)) to be submitted to the system are also given coordinates by the central module. Each Web host is assigned to a peer if the Web host's key falls into that peer's zone according to CAN's routing mechanism. When the inserting message of a Web host arrives at its destination peer, the peer invokes the crawling process on itself to crawl that Web host. The crawling process terminates itself when the crawling task is complete. In order to keep the retrieved Web data up to date, all the Web hosts will be periodically re-crawled by the crawlers.

According to the algorithm described so far, the relationship between crawlers and Web hosts is established in which a peer is responsible for crawling a set of Web hosts, at the same time one single Web host is only owned by one single crawler. In addition to this scheme, task replication can be utilized to achieve fault tolerance. But to avoid further complexity, in the following part of the paper, we don't replicate one Web hosts to multiple crawlers.

Upon the discovery of an inter-link, the crawler queries the central module for the key of the inter-link's host. On receiving the reply of the central module, the crawler then routes the inter-link to its destination crawler using the replied key as is demonstrated in Fig. 2 (c). To exchange the inter-link, we only need the key of the Web host not the address of the destination crawler. We just leave the job of finding the destination crawler to CAN's routing mechanism. This is because the set of the crawlers tends to be unstable. A crawler appears to be alive now may go offline a moment later with its zone and tasks taken by another crawler. The routing mechanism of CAN can easily cope with such changes. A cache is built in the crawler in order to reduce the number of queries to the central module. The method of batch exchanging can also be used to further optimize the process.

\subsection{Problem Formulations}

Based on the proposed system model, we formulate the problems we meet as follows.

Definition 3.1. Host Level Web Partition: Assuming that the set of the crawlers in the DWC system is $P=$ $\left\{p_{1}, p_{2}, \ldots, p_{n}\right\}$ and the set of the Web hosts is $R$, the system partitions $R$ into a set of subsets $B=\left\{b_{1}, b_{2}, \ldots, b_{n}\right\}$ so that $\left|b_{i} \cap b_{j}\right|<\delta, i=1,2, \ldots, n ; j=1,2, \ldots, n ; i \neq j$ and $b_{1} \cup b_{2} \cup \ldots \cup b_{n}=R, \delta$ is a small enough integer. The size of $B$ equals to the size of $P$ so that each $b_{i}$ can be assigned to a unique $p_{j}$. The above process is called the Host level Web Partition. It is symbolized as $F(P, R) . b_{i}$ is called a partition set.

Definition 3.2. Peer-Resource Distance: Assuming that the $i$ th $(1 \leq i \leq|P|)$ crawler named $p_{i}$ is responsible for a set of Web hosts $b_{p_{i}}=\left\{r_{p_{i}}^{1}, r_{p_{i}}^{2}, \ldots, r_{p_{i}}^{m}\right\}$; we define the peerresource distance (PRD) as the network distance between $p_{i}$ and $r_{p_{i}}^{t}(1 \leq t \leq m)$. The PRD can be symbolized as $L\left(p_{i}, r_{p_{i}}^{t}\right)$. The system's average PRD can be calculated through the following function: 


$$
A_{P R D}=\frac{\sum_{i=1}^{|P|} \sum_{t=1}^{\left|b_{p_{i}}\right|} L\left(p_{i}, r_{p_{i}}^{t}\right)}{\sum_{i=1}^{|P|}\left|b_{p_{i}}\right|}
$$

Definition 3.3. Inter-link and Intra-link: Assuming that a link (URL) is extracted from a Web page included in $b_{i}$, if the link points to a Web page also included in $b_{i}$, the link is called an intra-link; if the link points to a Web page that is not included in $b_{i}$, it is called an inter-link.

Definition 3.4. Communication Overhead: Assuming that the number of the inter-links is $E$; The number of inter-host links is $U$, the communication overhead of the system can be calculated through the following function:

$$
C_{\text {comm }}=\frac{E}{U}
$$

Definition 3.5. Load Variation: Assuming that the number of Web pages on the Web host $r_{p_{i}}^{t}(1 \leq t \leq m)$ is $P N_{r_{i}^{t}}$, crawler $p_{i}$ 's capacity is $C_{p_{i}}(1 \leq i \leq|P|)$; we define the relative load of a crawler as:

$$
R L_{i}=\frac{\sum_{t=1}^{\left|b_{p_{i}}\right|} P N_{r_{p_{i}}}}{C_{p_{i}}}
$$

The mean of each crawler's relative load is:

$$
\overline{R L}=\frac{\sum_{i=1}^{|P|} R L_{i}}{|P|}
$$

The load variation of the system equals to the Dispersion Coefficient of each crawler's load. It can be calculated through the following function:

$$
L V=\sqrt{\frac{\sum_{i=1}^{|P|}\left(R L_{i}-\overline{R L}\right)^{2}}{(|P|-1)}} / \overline{R L}
$$

Based on the above statement, we outline the problem we are facing: Given a set of crawlers $P$ and a set of Web hosts $R$, we want to establish a certain Web partition through $F(P, R)$ so that the PRDs, the communication overhead, and the load variation are kept low. The problem should be solved in a distributed manner since $P$ and $R$ are huge and subject to constant change.

\section{Network-Distance-Based Web Partition}

\subsection{Defining the Network Distance}

Most NCs are using network latency (equivalence with RTT) as the metric of the network distance. However, the network latency is not the only determining factor in the field of Web crawling.

In a typical download scenario, a crawler downloads
Web pages from a certain Web host in pipeline through http persistent connections, so that multiple Web pages can be downloaded in one TCP connection. In order to perform politely, the crawler should only keep one TCP connection to the Web host at one time. After each download, there must be a politeness wait time interval.

According to the above process, the total download time of a Web page (or several pages under HTTP pipelining) is determined by three factors: (1) the round trip time (RTT) cost by initializing a (or several in pipeline) HTTP request; (2) the actual download time (DLT) which is the time gap between the arrival of the first byte of the Web page (or several pages in pipeline) and the arrival of the last byte of the Web page. (3) the politeness wait time (PWT) interval which is a pre-configured constant value. The RTT is decided by the network latency, while the DLT is decided by the bandwidth. Thus the ratio of DLT and RTT largely depends on the crawler's residing environment. For example, in a low latency low bandwidth environment, the DLT may be much bigger than the RTT; on the contrary, in a high latency high bandwidth environment the RTT stands out.

In our system, the network distance between a crawler and a Web host equals to the combination of the median RTT $\left(A_{R T T}\right)$ and the average DLT $\left(A_{D L T}\right)$ between the crawler and the Web host (Formula 6). The two values are obtained through HTTP-based probing during which several HTTP sessions are conducted between the crawler-host pair and the corresponding time costs are recorded. The inter-crawler probing is implemented by simulating the HTTP download process.

$$
N D=A_{R T T}+A_{D L T}
$$

$A_{R T T}$ equals to the median RTT of multiple HTTP HEAD tests (we call it the http-ping). This value is very similar to that of ping. To calculate $A_{D L T}$, we assume that during the probing, $K$ pages are downloaded in pipeline; the size of each downloaded page is $S_{i}, 0 \leq i<K$; the average size of each page on the Web host is $\bar{S}$; the DLT equals to $T$; then the $A_{D L T}$ is calculated as follows:

$$
A_{D L T}=(\varepsilon \times \bar{S}) /\left(\frac{\sum_{i=0}^{K-1} S_{i}}{T}\right)=(\varepsilon \times T \times \bar{S}) / \sum_{i=0}^{K-1} S_{i}
$$

$\varepsilon$ is the actual number of pages downloaded in pipeline during the real crawl. The formula means that the $A_{D L T}$ equals to the time cost for downloading $\varepsilon$ Web pages in pipeline.

According to the above statement, PRD $L\left(p_{i}, r_{p_{i}}^{t}\right)$ in Definition 3.2 equals to the network distance (ND) between $p_{i}$ and $r_{p_{i}}^{t}$. According to Definition 2, each crawler-host pair possesses a PRD. Therefore the number of PRDs in the system is $\sum_{i=1}^{|P|}\left|b_{p_{i}}\right|=|R|$.

\subsection{Combining NC and CAN}

Inspired by the Topologically-aware CAN [18] and NC- 


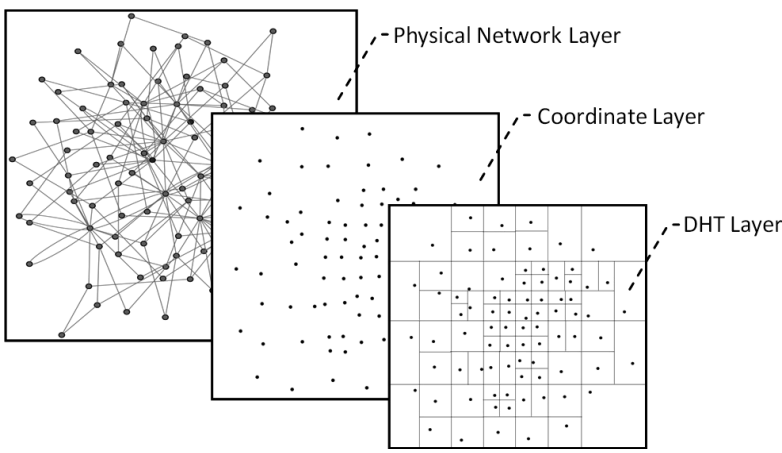

Fig. 3 Mapping from network positions to the DHT overlay.

CAN [19], we choose to reduce the PRDs using the Network Coordinate System (NC) [20]-[23]. The central module accordingly can be implemented as a set of peers distributed across the internet assembling a NC. The network coordinates of the crawlers and the Web hosts are calculated according to the network distances measured by the landmarks.

The mapping from network positions to DHT is demonstrated by Fig. 3. The first step is to map the nodes on the network layer to the network coordinates on the coordinate layer. This task is performed by the $\mathrm{NC}$ of the central module. The second step is to map the network coordinates to the DHT layer (CAN space). The network coordinates of the crawlers are used as DHT's IDs; on the other hand, the network coordinates of the Web hosts are used as keys. The number of dimensions in the CAN space is determined by the number of dimensions of the network coordinates. Each crawler queries the central module for new network coordinate after each time interval (24 hours). If a crawler's new coordinate shifts too far away from the central coordinate of the crawler's zone (the length of the zone's diagonal can be used as the threshold), then the crawler automatically leaves the DHT and re-join using the new coordinate as its ID. During the re-join process, the crawling tasks assigned to the crawler still runs. According to Sect. 3, the Web hosts are re-submitted to the DHT during their re-crawl. Before the submission, each Web host's network coordinate is renewed, so that they can be assigned to the latest optimal crawler.

To combine NC and CAN, there are several main problems we have to face. First, the upper and lower bounds of the CAN space is different from that of the network coordinate space (NCS). Because the metric of the network coordinate equals to the network distance on the real network, the network coordinate space can be logically infinity. On the contrary, the CAN space should be a limited space with upper and lower bounds on each dimension. In other words, the CAN space is only a subspace of the NCS. Moreover, in order to achieve a balanced load distribution, the node density in the CAN space should be high enough.

According to our analysis to the distribution of nodes on the NCS, most nodes concentrate in one area while the rest lies far away from the majority. In our system, we use

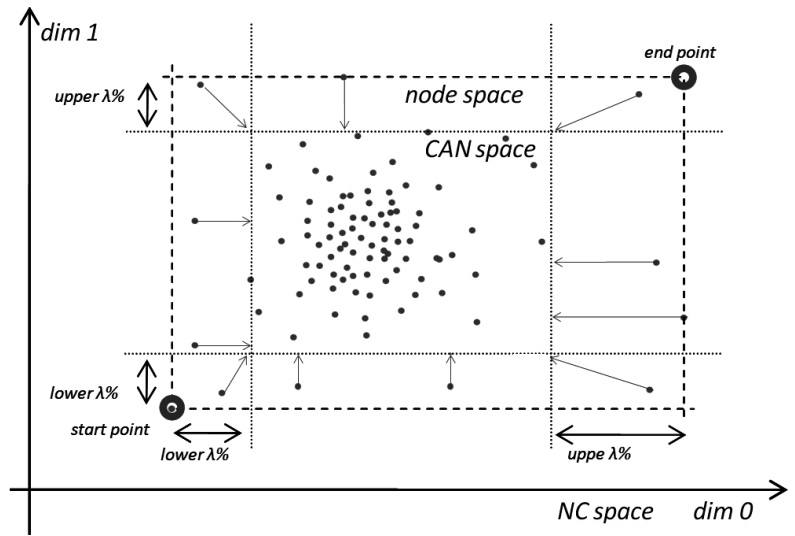

Fig. 4 Allocating the CAN space from the NC space.

a simple method (Fig. 4 shows a demonstration on a $2 \mathrm{~d} \mathrm{NC}$ space) to allocate the CAN space from the NCS. The set of coordinates (nodes on the NCS) used to allocate the CAN space is from the historical records of known Web hosts' network coordinates. (1) first, we find the lowest and the highest coordinate values on each dimension. By combining all the lowest coordinate values we get a "lowest" point in the NC space called the start point. Meanwhile, by combining all the highest coordinate values we get a "highest" point called the end point. Now all the nodes are included in the cube space (we call the node space) between the start point and the end point. (2) Then we push the lower bound of the node space on each dimension upward until $\lambda \%$ nodes are left outside. At the same time, we push the upper bound on each dimension downward until $\lambda \%$ nodes are left outside. The cube space within the new bounds are the CAN space we need. (3) Each node left outside during (2) is moved to the boundary closest to it. When a new node (for example a crawler or a Web host) joins the system, its final coordinate can be calculated as follows. Assuming that in the n-dimensional NCS, the lower bounds of the CAN space are $l_{0}, l_{1}, \ldots, l_{n-1}$, while the upper bounds are $h_{0}, h_{1}, \ldots, h_{n-1}$; the new node's network coordinate given by the NC is $\left(t_{0}, t_{1}, \ldots, t_{n-1}\right)$; then the new node's final coordinate on each dimension is $t_{i}^{\prime}=\left(l_{i} \leq t_{i} \leq h_{i} ? t_{i}:\left(l_{i}>\right.\right.$ $\left.\left.t_{i} ?\left(l_{i}+\delta\right):\left(h_{i}-\delta\right)\right)\right), 0 \leq i<n, \delta$ is a randomly generated small value.

Second, the network positions of the crawlers and the Web hosts subject to change. Because the RTT between each crawler-host pair is changing 24 hours a day, the result of a single probing is unable to conclude a RTT value with guiding significance. However, the network distance's daily trends subjects to little change. And J. Exposto [24] proposes a method to conclude stable RTTs with minimum number of daily probing. The idea can also be applied to the network distance. With the stable network distances, the network coordinates don't have to change constantly. Even if the network coordinate of a crawler changes, as long as the shift remains smaller than the threshold, it is not necessary to replace the old coordinate with the new one. In our system, the central module can re-calculate the crawlers' and 
the Web hosts' network coordinates and reassigns the new coordinates to them. To avoid the confusion caused by the reassignment, each Web host's coordinate is reassigned during the time gap of the re-crawl processes. Upon the discovery of a new Web host, the central module either finds the key value in its pre-reserved database or measures the Web host's network position through NC instantly. On the other hand, each crawler's coordinate is reassigned by leaving and re-joining the DHT.

One more issue is that the DLTs cannot be accurately estimated by the NC. Therefore, we only use NC to estimate the locations in the RTT space. For the DLTs, we adopt a neighbor-table-based redirect method to try to approach the proximity of the DLT. When a Web host $\mathrm{W}$ is assigned to a crawler $C$ according to the NC-CAN scheme, $W$ is not immediately be crawled by $C$. instead, $C$ sends queries to a portion of its neighbor (may not limited to the direct neighbors). On receiving the query, each neighbor conducts a series of short time probing, to test the network distance (both RTT and DLT) between itself and $W$. The crawler who possesses the shortest network distance in the above session finally takes over $W$. One thing to note is that $W$ is not permanently assigned to a certain crawler. The choice may change in the next re-crawl.

Through the process given above, we implement a network-position-aware DHT. From the overall point of view, the whole collection of crawlers and Web sites is partitioned according to their network positions. Thus the goal of reducing the peer-resource distance is achieved. We call the above method the network-distance-based Web partition (ND-based partition).

\section{Link-Based Web Partition}

In addition to the network overhead generated by the downloading processes, there are other network communications among the servers in the system. These kinds of communications can be classified as two types:

- System level communications: The communications caused by the basic DHT operations.

- Business level communications: The intra-system communications generated by the crawling processes. They serve the roles to ensure the crawling functionalities.

Since the DHT operations only generates small messages and are only performed on rare occasions, the fundamental cause of the business level communications is the interlink exchanging. Assuming that the total number of URLs to be downloaded by our system is $N_{U R L}$, then in order to download these URLs, the maximum number of communications conducted by the crawlers is $C_{\text {download }}=N_{U R L}$. We also assume that the number of inter-links is $N_{\text {inter-link}}$; the average number of hops caused by routing one inter-link is HOPS; each inter-link may be discovered $R$ times during the crawl. Then in order to exchange these inter-links, the number of communications conducted by the crawlers is
$C_{\text {exchange }}=N_{\text {inter-link }} \times H O P S \times R$. According to our experience, in a system with more than 100 peers, HOPS $\approx 3$; and $N_{\text {inter-link }} \approx N_{U R L} / 100$ and $R \approx 10$. Then $C_{\text {exchange }} \approx$ $0.3 \times C_{\text {download }}$. Although both URL downloading and interlink exchanging can be batch processed, the equation shows that $C_{\text {exchange }}$ is quite a large number that can't be ignored. The problem becomes even worse when the DWC system is using rented private network to transmit inter-crawler communications, because you have to pay for the traffic.

In this section, we focus on reducing the business level communications by reducing the number of inter-links and the length of the inter-links' routing path. Inspired by the concept of NC, we solve the problem by mapping the relative positions of the Web hosts on the link topology to the coordinates in a unique logical space. The distance between different coordinates equals to the link distance between the corresponding Web hosts. The Web hosts are submitted to the system using the coordinates as their keys. In the following literatures, we describe in detail how the above method is achieved.

\subsection{The Link Coordinate}

First, we give the following definitions and formulas:

Definition 5.1. Host level link graph (link graph): We symbolize the host level link graph as $G(V, E) . V$ is the set of nodes on the graph. Each node represents a unique Web host on the Internet. $E$ is the set of directed edges on the graph. An edge connects two nodes only if there is at least one link between them. The direction of the edge depends on the direction of the links.

Definition 5.2. Cost of the edge: A cost is assigned to each edge on $G(V, E)$ Assuming there's an edge from node $v_{x}$ to node $v_{y}$; node $v_{x}$ represents Web host $x$ and node $v_{y}$ represents Web host $y$; the number of links from host $x$ to host $y$ is symbolized as $l_{(x, y)}$; the edge is symbolized as $e_{\left(v_{x}, v_{y}\right)}$, then the cost of the edge $e_{\left(v_{x}, v_{y}\right)}$ can be calculated through the following function ( $c$ is a constant to keep the value big enough):

$$
f\left(e_{\left(v_{x}, v_{y}\right)}\right)=\frac{c}{l_{(x, y)}}
$$

Definition 5.3. Link distance: Assuming on $G(V, E)$ the shortest path from node $v_{x}$ to node $v_{y}$ consists of edges $e_{\left(v_{x}, v_{x_{1}}\right)}, e_{\left(v_{x_{1}}, v_{x_{2}}\right)}, \ldots, e_{\left(v_{x_{n-1}}, v_{x_{n}}\right)}, e_{\left(v_{x_{n}}, v_{y}\right)}$ and the shortest path from node $v_{y}$ to node $v_{x}$ consists of edges $e_{\left(v_{y}, v_{y_{1}}\right)}, e_{\left(v_{y_{1}}, v_{y_{2}}\right)}, \ldots, e_{\left(v_{y_{m-1}}, v_{y_{m}}\right)}, e_{\left(v_{y_{m}}, v_{x}\right)}$, then the link distance $d(x, y)$ between the two nodes can be calculated through the following formulas:

$$
\begin{aligned}
& \overleftarrow{d}(x, y) \\
& \quad=\left\{\begin{array}{c}
\infty, \text { if } y \text { is unreachable from } x \\
f\left(e_{\left(v_{x}, v_{y}\right)}\right), \text { if } x \text { and } y \text { are directly linked } \\
f\left(e_{\left(v_{x}, v_{x_{1}}\right)}\right)+f\left(e_{\left(v_{x_{n}}, v_{y}\right)}\right)+\sum_{i=2}^{n} f\left(e_{\left(v_{x_{i-1}}, v_{x_{i}}\right)}\right) . \text { else }
\end{array}\right.
\end{aligned}
$$




$$
\begin{aligned}
& \overleftarrow{d}(y, x) \\
& =\left\{\begin{array}{c}
\infty, \text { if } x \text { is unreachable from } y \\
f\left(e_{\left(v_{y}, v_{x}\right)}\right), \text { if } y \text { and } x \text { are directly linked } \\
f\left(e_{\left(v_{y}, v_{y_{1}}\right)}\right)+f\left(e_{\left(v_{y_{n}}, v_{x}\right)}\right)+\sum_{i=2}^{m} f\left(e_{\left(v_{y_{i-1}}, v_{y_{i}}\right)}\right), \text { else }
\end{array}\right. \\
& d(x, y)=\min (\overleftarrow{d}(x, y), \overleftarrow{d}(y, x))
\end{aligned}
$$

Definition 5.4. Relative error: Assuming the Euclidean distance between the link coordinates of node $v_{x}$ and node $v_{y}$ is $d^{\prime}(x, y)$, and the link distance between node $v_{x}$ and node $v_{y}$ is $d(x, y)$, then the relative error of the node pair $(x, y)$ equals to:

$$
\operatorname{error}(x, y)=\frac{\left|d^{\prime}(x, y)-d(x, y)\right|}{\min \left(d^{\prime}(x, y), d(x, y)\right)}
$$

Definition 5.5. Error minimization: Given a link graph $G(V, E)$, every node in $V$ is mapped to a unique link coordinate in a limited link coordinate space (LCS) so that the relative error of any node pair is minimized.

The host level link graph is collected from the link statistics recorded in the previous crawl. The link graph is not necessarily a connected graph, as the crawl usually begins with a large number of irrelevant seed URLs. Different from the URL level link graph, the host level link graph consists of much fewer nodes and edges than the former. This makes the graph much easier to process. The cost of each edge on the graph is defined by Formula 8 to ensure that the two Web hosts are closer to each other on the link graph if there are more links between them.

Based on the host level link graph, we have to calculate the link distance between any two nodes. Here we use the shortest path to define the link distance. The link distance is an undirected value. It is decided to be undirected, because all we care about is the number of communications caused by the inter-links not the inter-links' directions. However, not all nodes are reachable from any node on the link graph. In practical, if the link distance between two nodes equals to infinity according to Formula 11 (we call these link distances the unknown link distances (ULDs)), then the distance is undefined.

After the calculation we get a matrix of link distances called link distance matrix (LDM), the link coordinates are generated according it. We recognize the problem of generating the link coordinates as an error minimization problem. The concept is defined by Definition 5.5. In practice, as the existing NCs are also using error minimization to generate the network coordinates, they can be utilized to achieve our goal. In other words, NC is used here not to predict the network distance but only to perform the error minimization.

The generated coordinates are called the link coordinates. The new logical space resided by the link coordinates is called the link coordinate space (LCS). The Web hosts are submitted to the system using their link coordinates as keys.

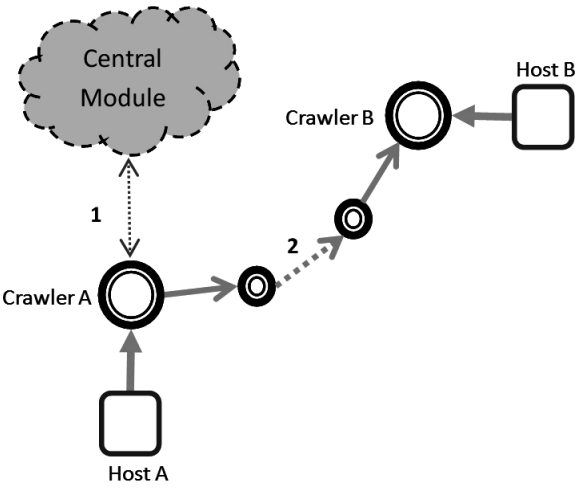

Fig. 5 The communication process under link-based partition.

On the other hand, the crawlers are also given specially calculated link coordinates (we call the avatars, the method is explained in 5.2) so that both the crawlers and the Web hosts are mapped to the same LCS.

The allocation method proposed in 4.2 is used to allocate a CAN space (DHT space) from the LCS. By inserting the crawlers and the Web hosts into this new CAN space, the Web hosts located closely on the link topology can be maintained on the same crawler. As a result, the links between these Web hosts can be processed in the crawler's local memory, avoiding the network communications. Moreover, even to the inter-links that have to be exchanged among the crawlers, the length of the routing path is well shortened as the CAN overlay is built according to the proximity on the link topology. We call the above method the link-based partition.

With crawlers and Web hosts all mapped to the LCS, the communication process for inter-link exchanging is demonstrated by Fig. 5. To transfer an inter-link to its destination at most 2 main stages are involved. Assuming that a number of links pointing to Host B are discovered by Crawler A, then:

1) Crawler A queries the Central Module for the link coordinate of the inter-link's host (Host B).

2) Crawler A route the inter-link using Host B's link coordinate as the key on the DHT.

A cache storing the known host's link coordinates can be built on Crawler A to avoid stage 1. Both on Stage 1 and 2, messages can be batch-transferred to reduce the communication overhead.

\subsection{Mapping the Crawlers to the Link Space}

In order to make the DHT work, the crawlers and the Web hosts should share a common logical space. However, as the crawlers are not linked to any Web hosts, the crawler's link coordinates cannot be generated using the way we mentioned in 5.1. Here we assign each crawler a predesigned link coordinate called the avatar. We have developed 3 ways to create the avatar:

- Random generation: The system randomly generates 
a link coordinate for each crawler.

- Random selection: For each crawler, the system randomly selects a known Web host's link coordinate and assigns it to the crawler.

- ND-based selection: For each crawler, the system selects a Web host which is located closest to the crawler in the network coordinate space and assigns the Web host's link coordinate to the crawler.

The avatars generated using the first method may not be in compliance with the distribution of the Web hosts. As a result, the set of the Web hosts may not be evenly partitioned by the crawlers. The second and third methods solve the problem but introduce the uneven partition of the LCS. In practice, different method should be chosen according to different needs.

\section{Distributed Multi-Objective Web Partition}

In this section we combine the ND-based partition and the link-based partition to achieve a distributed multi-objective Web partition. As the system is using Content Addressable Network (CAN), we adopt the two basic approaches of CAN to establish this combination: multi-space and multidimension.

\subsection{Multi-Space}

In this approach, the system uses two logical spaces: one is the network coordinate space (NCS), another one is the link coordinate space (LCS). The main concept is to crawl the Web hosts according to the proximity of the NCS, while communicate the crawlers according to the proximity of the LCS.

When a crawler joins the system, it separately joins the two logical spaces. When a Web host is submitted to the system, it is also separately inserted to the two logical spaces. As a result, a Web host may be maintained by two different crawlers in the two logical spaces. To avoid this ambiguity, the crawler who owns the Web host in the NCS (call the crawler as the Web host's NCS crawler) records the address of the crawler who owns the Web host in the LCS (call the crawler as the Web host's LCS crawler), and vice versa. The Web host's NCS crawler is actually in charge of crawling the Web host.

The communication process is shown in Fig. 6. To simplify the description, in the following literature we assume that the link coordinates of the involved Web hosts are known to the corresponding crawlers. As an example, we assume that initially the system assigns Host A to Crawler $\mathrm{A}$ and assigns Host B to Crawler B. The communication process involves 4 stages:

1) Crawler $A$ discovers an inter-link in Host $A$ which points to Host B.

2) Crawler A pushes the inter-link to Host A's LCS crawler - Crawler A'.

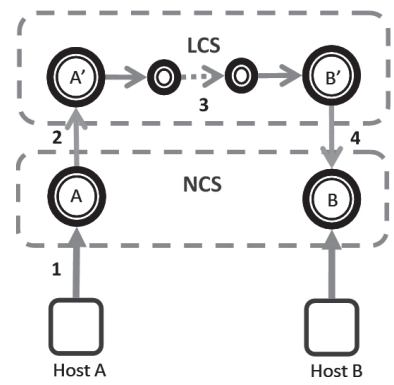

Fig. 6 The communication process under multi-space approach.

3) On receiving the pushed inter-link, Crawler $A^{\prime}$ extracts the hostname of Host B from the inter-link, and routes the inter-link according to Host B's link coordinate in the LCS. According to our assumption, the inter-link should be routed to Crawler B.

4) Crawler B' pushes the inter-link to Host B's the NCS crawler - Crawler B.

The process involves two more communications on stage 2) and 4) than the method described in Sect. 4. On Stage 2), 3) and 4) messages can be batch-transferred to reduce the number of communications.

This approach takes the benefits of both the NCS and the LCS. Moreover, as the LCS only bears the task of communication, the link coordinates of the Web hosts can be re-calculated and re-assigned even when they are still being crawled without interfering the downloading process. The weak point of the approach is that the system has to maintain two different and mutually connected logical space. Moreover, the two more push operations cause more communication overhead.

\subsection{Multi-Dimension}

In this approach, the system uses one single logical space with relatively large number of dimensions. Assuming that a Web host or a crawler's network coordinate is $\left(x_{0}, x_{1}, \ldots, x_{n}\right)$ and its (or its avatar's) link coordinate is $\left(y_{0}, y_{1}, \ldots, y_{n}\right)$, then its coordinate in the system is $\left(x_{0}, \ldots, x_{n}, y_{0}, \ldots, y_{n}\right)$ or $\left(y_{0}, \ldots, y_{n}, x_{0}, \ldots, x_{n}\right)$ which is the combination of the two coordinates. Whenever an event such as joining of a crawler or inserting of a Web host happens, the system simply follows to rule of CAN to handle it. When there's an inter-link to be exchanged among crawlers, the discovering crawler routes the inter-link directly on the CAN overlay.

This approach avoids the inconsistency problem caused by the multi-space approach. With more dimensions, the CAN overlay can shorten the average length of the routing paths, thus reducing a considerable amount of communication overhead. However, this approach can only achieve a compromised performance compared with the ND-based partition and the link-based partition. One optimization alternative for this method is to use the ND-based selection when assigning link coordinates to the crawlers. In addi- 
tion, to avoid confusion, the link coordinate of each Web host can only be reassigned during the time gap of the recrawl processes.

\section{Experiment}

\subsection{Experiment Setups}

To prove the effectiveness of the link-based partition, we use 5 link topology datasets in the simulations. The first dataset is a subset of a larger dataset collected and published by Sogou Labs [25] in the year 2008. The dataset contains 1600 Web sites, 1447893 URLs, 3382 edges and 15751 inter-host URLs. The dataset is called the real dataset. Its host level link graph is very sparse. Although the average out-degree of each node is 2, 90\% out-degrees are less than 2 . The other four datasets are simulated graphs based on the Barabási \& Albert (BA) model and the random model. Each host in these dataset is assigned 100 URLs. Thus each simulated dataset has $1600 \times 100$ URLs. According to the distribution of the out-degree in the real dataset, we set the average out-degree of the simulated datasets to 1 and 2 . The visualizations of the datasets are shown in Fig. 7. And the specific parameters of the datasets are outlined in Table 1.

As the existing NCs have fully proved the effectiveness of the network coordinates, we only use one widely accepted dataset called king published by p2psim [26]. The short coming of the king dataset is that it doesn't contain the download time (DLT). As a result, we treat the latencies in the dataset as the network distance. Because the dataset contains 1740 nodes, we randomly select 140 nodes as the crawlers. The rest 1600 nodes are treated as Web hosts. The output of the ND-based partition is not separately presented in the following literature. Instead, it is given in the output of the multi-objective partition. We choose to do so because the Multi-space approach's PRD performance is exactly the same as that of the ND-based partition.

Because the simulations involve both network distance and link topology, we combine each link topology dataset with the king dataset by randomly mapping the 1600 king nodes to the 1600 nodes on each link topology dataset. As a result, the combined datasets have two layers: the link layer and the network layer, which matches the situation of the real Internet.

The nodes in each link topology dataset are mapped to 3-d link coordinates using the method proposed in Sect. 5. We use the Vivaldi program [27] as the tool to perform the error minimization. We also use Vivaldi to generate the 3-d network coordinates of the king dataset. The network coordinates are transformed using the method mentioned in 4.2 with $\lambda=2$. The results are shown in Fig. 8. The relative errors of the error minimization are shown in Fig. 9. We also recognize that the distributions of the coordinates shown in Fig. 8 reflect the topologies in Fig. 7. On one hand, in Fig. 7, Web hosts in (c) and (e) show high connectivity. These topologies generate evenly distributed coordinates in Fig. 8 (c) and (e). On the other hand, Web hosts in Fig. 7 (b)

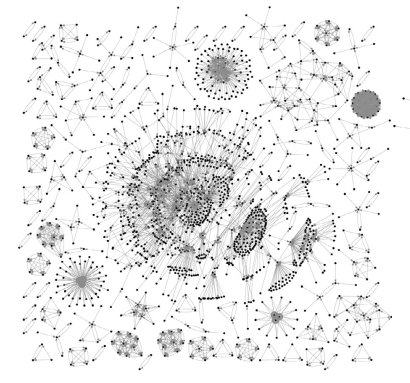

(a) real

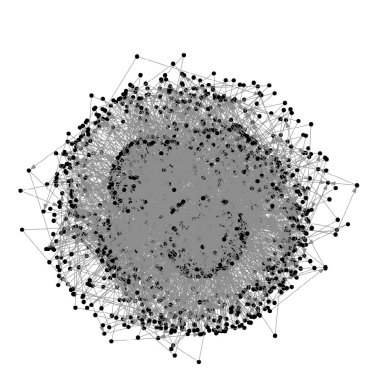

(c) BA_edge 2

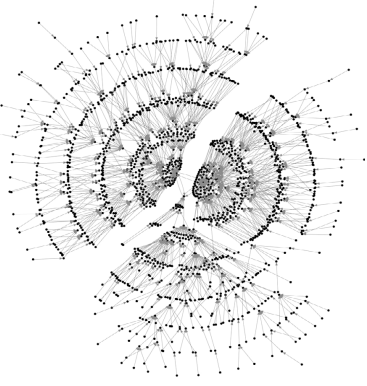

(b) BA_edge_1

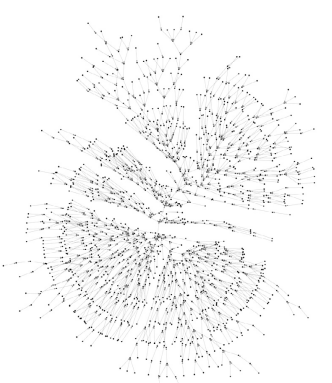

(d) Rand_edge_1

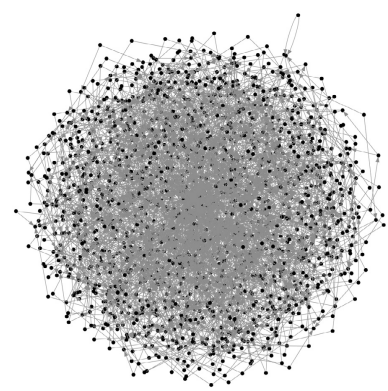

(e) Rand edge 2

Fig. 7 Visualizations of the link topology datasets.

Table 1 Parameters of the link topology datasets.

\begin{tabular}{lllllll}
\hline Dataset Name & Model & $\begin{array}{c}\text { Web } \\
\text { hosts }\end{array}$ & URLs & $\begin{array}{l}\text { Inter- } \\
\text { host } \\
\text { URLs }\end{array}$ & Edges & $\begin{array}{l}\text { Avg } \\
\text { Out- } \\
\text { degree } \\
\text { (edge) }\end{array}$ \\
\hline Real & Real & 1600 & 1447893 & 15751 & 3382 & 2 \\
\hline BA_edge_1 & BA & 1600 & 160000 & 1600 & 1600 & 1 \\
\hline BA_edge_2 & BA & 1600 & 160000 & 3200 & 3200 & 2 \\
\hline Rand_edge_1 & Random & 1600 & 160000 & 1600 & 1600 & 1 \\
\hline Rand_edge_2 & Random & 1600 & 160000 & 3200 & 3200 & 2 \\
\hline
\end{tabular}

and (d) show low connectivity. Accordingly, coordinates in Fig. 8 (b) and (d) tends to gather in small clusters. The real dataset in Fig. 7 (a) and Fig. 9 (a) appears to be a combination of the above two situations. In addition, the King dataset in Fig. 8 (f) shows 3 major clusters.

Based on the datasets described above, we conduct a series of DHT simulations involving peer joining and resource insertion. All the peers are assumed to have the same 


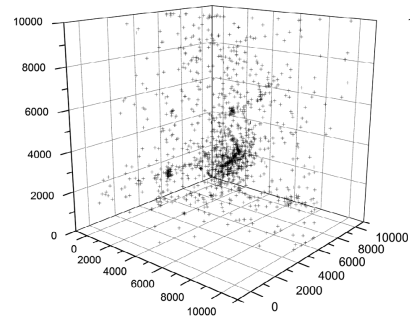

(a) real

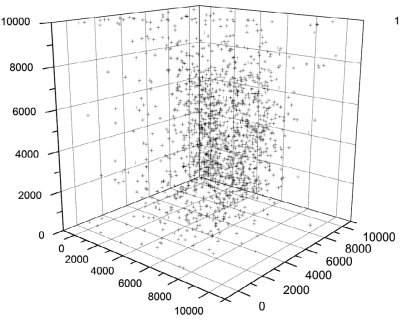

(c) BA_edge_2

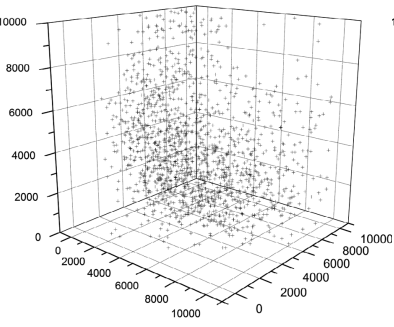

(e) Rand_edge_2

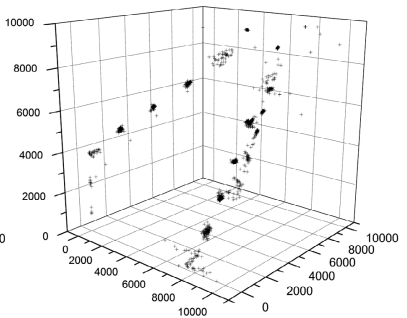

(b) BA_edge_1

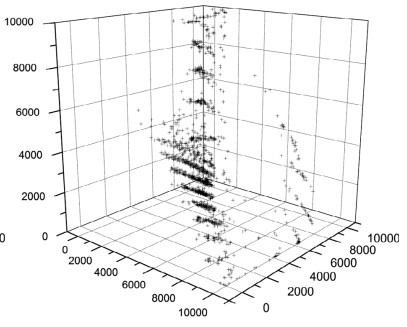

(d) Rand_edge_1

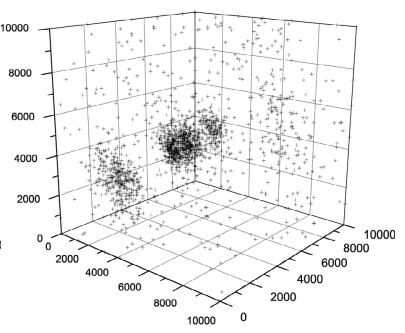

(f) King
Fig. 8 The coordinate generated through error minimization.

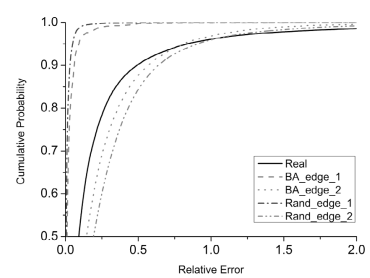

(a) link coordinates

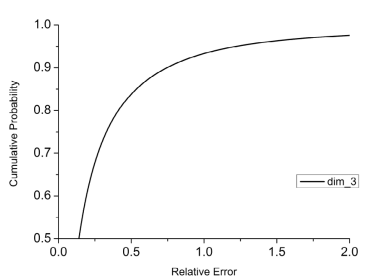

(b) network coordinates (King)
Fig. 9 Relative error (CDF).

capacity. We simulate the inter-link exchanging scenarios by letting each crawler conduct a series of inter-link exchanging according to the link topology. The exchanging of each inter-link is performed only once. In the real world, each inter-link may be exchanged many times due to the duplication on the Web. Since such duplication can't affect the overall trend, we don't add duplications to the simulations. In addition, since batch exchanging causes the same proportional changes to all methods, we don't adopt batch exchanging either.

The proposed methods are compared with the method of Apoidea [11]. The Apoidea system is a DHT-based DWC system with all crawlers joining a Chord [28] ring. To reduce the peer-resource distance, we introduce an experimental finger-table-based redirect method for Chord (we call Chord redirect). In this method, when a Web host is assigned to a peer, the peer asks all peers in its finger table to measure the network distance to the Web host. The peer with the shortest network distance is chosen to hold the Web host.

\subsection{Reducing the Communication Overhead}

In this section, we concentrate on the communication overhead and ignore the influences of the crawler-crawlee network distance. The simulations are run on the 5 link topology datasets and in 14 different system scales (from 10 crawlers to 140 crawlers). In the simulations, 3 methods are compared:

1) CAN link-based: It's the case of link-based partition. The avatars are selected using Random selection.

2) CAN: In this method, we assign SHA1 hashed coordinates (IDs) to the crawlers and the Web hosts.

3) Chord: In this method, we assign SHA1 hashed IDs to the crawlers and the Web hosts. Without the consideration of network distance, we do not use the redirect method here.

The results are shown in Fig. 10. For each link topology dataset, 3 columns of figures are presented. From left to right, the 3 columns respectively present:

1) The communication overhead of the system (CO)

2) The number of the inter-links exchanged (IE)

3) The average length of the routing paths (RP)

In the 1st column, CAN has the highest communication overhead, meanwhile, CAN link-based has the lowest. All 5 datasets show the same pattern proving the new method's superiority. Because the communication overhead of a DHT-based DWC system is caused by the number of the inter-links and the length of the inter-links' routing path. To gain a further conclusion, the statistics of the two factors are shown in the 2nd and 3rd figures.

In the 2nd column, the number of inter-links is divided by the number of inter-host links in the corresponding dataset. As the inter-links are all from the inter-host links, the value labeled on the vertical bar should be lower than 1.0. The SHA1-based methods (CAN and Chord) generate almost the same amount of inter-links. Numerically, the vertical values are very close to 1.0 , which means most of the links have become the inter-links. On the other hand, the vertical values of CAN link-based are always lower than that of CAN and Chord. However, this value grows rapidly when the system scale increases. This is because the correlation between the partition sets becomes higher and higher as the number of crawlers increases. Therefore more and more links become inter-links. This trend is inevitable to any method. To our experience, in order to avoid a large 

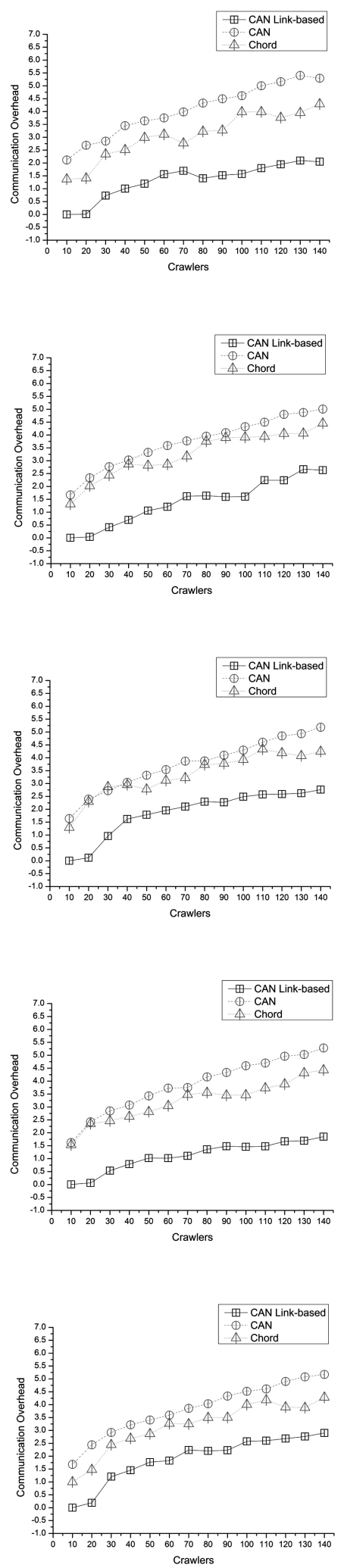
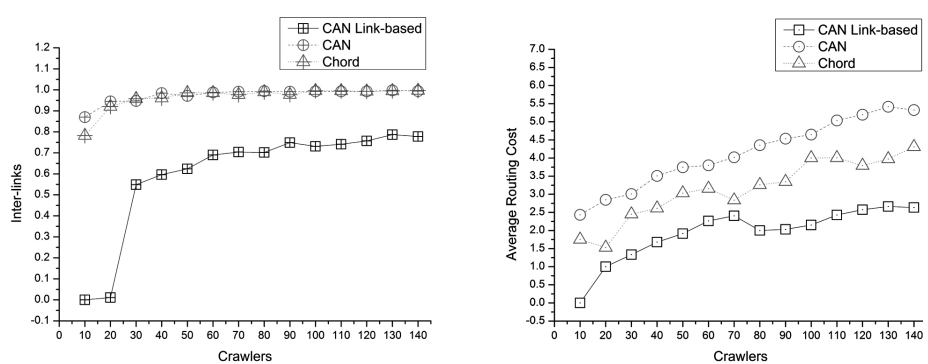

(a) real (CO, IE, RP)
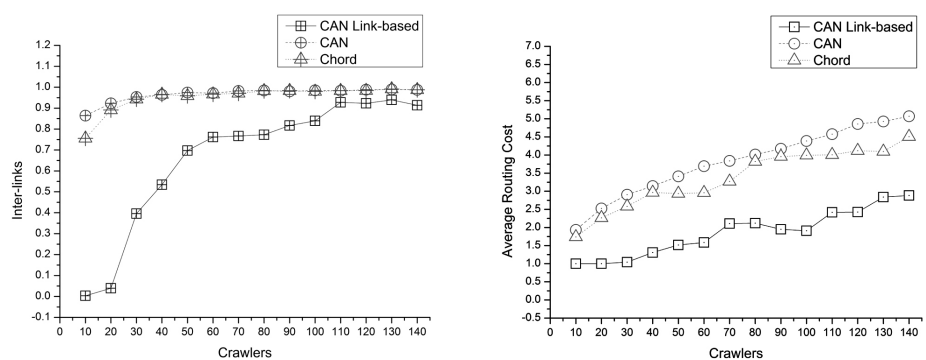

(b) BA_edge_1 (CO, IE, RP)
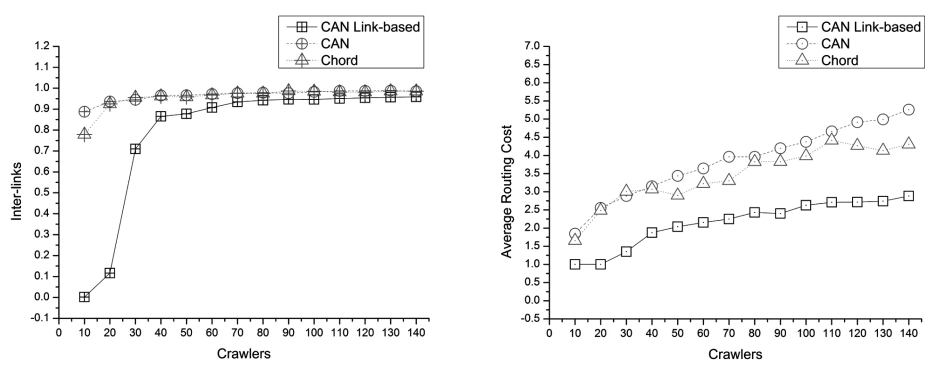

(c) BA_edge_2 (CO, IE, RP)
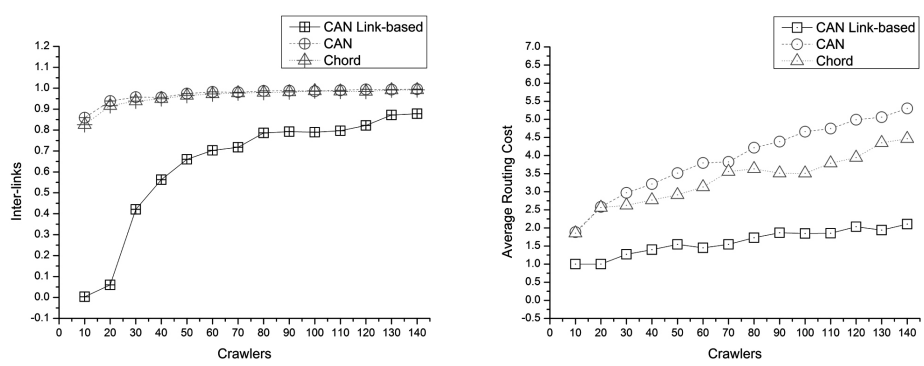

(d) Rand_edge_1 (CO, IE, RP)
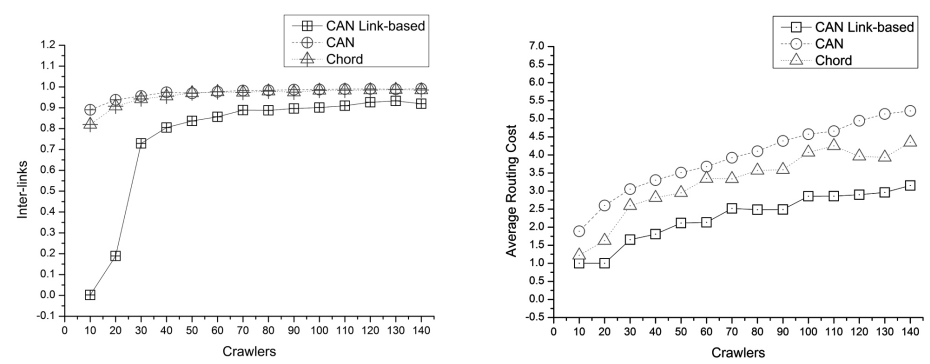

(e) Rand_edge_2 (CO, IE, RP)

Fig. 10 Comparisons on communication overhead. 
amount of inter-links, the ratio between the number of Web hosts and the number of crawlers should preferably be bigger than 10 .

In the 2nd column, all 3 curves tend to intersect when system scale reaches 140 except for the real dataset. This is because the real dataset is a non-connected graph while the others are not. This characteristic indicates that, to the real dataset, if the partition algorithm is well enough, many partition sets won't necessarily have inter-links connected between each other. For the rest datasets, CAN link-based performs a little worse when there are more inter-host links within the dataset (BA_edge_2, Rand_edge_2). Under these datasets, the curve of CAN link-based become very close to that of Chord when system scale exceeds 70 . This indicates the current link coordinate algorithm is not very efficient to a connected graph with a relatively large number of edges.

In the 3rd column, the number of hops during the DHT routing is used to evaluate the routing cost. The vertical value represents the average cost of each DHT routing during the simulation. On one hand, CAN has longer average routing cost than Chord. This reflects the theoretical routing length of Chord and CAN: $O(\log N)$ and $O(d \sqrt[d]{N})(d=3)$. Under the same system scale, CAN has longer routing path than that of Chord. As the system scale grows, the growth rate of CAN is also higher than that of Chord. It can clearly be seen that simply using the original CAN [17] gives no benefit to the system. On the other hand, although using CAN, CAN link-based break the above rule, and shows the shortest routing path. We believe that it is the concept of link coordinate that makes all the difference.

We conclude that the proposed method called CAN link-based shows its superiority in the fields of inter-links and routing cost. As a result, the method generates the lowest communication overhead. On the other hand, because CAN and Chord generate the same number of inter-links, the routing cost become the determinants of winning and losing. Therefore, Chord outperforms CAN, as it has shorter routing path.

\subsection{Distributed Multi-Objective Web Partition}

In this section, we add the network latencies to the simulations. Accordingly, the proposed distributed multi-objective Web partition methods are chosen to be compared with the Apoidea method. Here, we apply the finger-table-based redirect to the Chord ring. To push the method to its limit, we put no restrictions on the number of redirections. This option may lead to higher communication cost when inserting the Web hosts, although cost on inserting is not included in the results. In the simulation, 3 new methods are added:

1) CAN Multi-sp: This is the case of the multi-space approach described in 6.1.

2) CAN Multi-dim: This is the case of the multidimension approach described in 6.2.

3) Chord Redirect: This is the case of the finger-tablebased redirect.
For the link coordinates in 1) and 2), ND-based selection is used to find the crawlers' avatars. The results are shown in Fig. 11. For each link topology dataset, 3 columns of figures are presented. Different from 7.2, here we evaluate the system's performance on 3 aspects. From left to right, the 3 columns respectively present:

1) The communication overhead of the system (CO)

2) The average PRD of the system (AP)

3) The load variation of the system (LV)

In the 1st column, CAN Multi-dim has the lowest communication overhead. Chord Redirect and Chord performs almost the same. CAN Multi-sp doesn't perform well. This negative result is caused by the two more pushes as describe in 6.1. According to our previous analysis in 7.2, the average routing cost in the LCS is up to no more than 3 . Compared with this cost, the 2 pushes generate at least $2 / 3$ more communication overhead. Moreover, the number of pushes is proportional to the number of the inter-links generated by the ND-based partition. As the ND-based partition doesn't take the link topology into consideration, the number of pushes will be very high. To demonstrate this problem, we add a curve of CAN Multi-sp without the cost of the pushes. In all cases, this curve is always lower than all other curves. We also add a curve of CAN ND-based. In all cases, this curve is always among the highest ones. Although the output is not encouraging, the LCS in CAN Multi-sp should not be simply abandoned. Without the LCS, CAN Multi-sp will transform into CAN ND-based. In the cases of real, BA_edge_1 and Rand_edge_1, CAN Multi-sp still outperforms CAN ND-based. But, in the cases of BA_edge_2 and Rand_edge_2 where the number of inter-links is high, CAN Multi-sp loses all its advantages. Such fact indicates that CAN Multi-sp may still stand a chance in a sparse link topology.

In the 2nd column, all methods share the same dataset of network distance. As a result, CAN Multi-sp, CAN, Chord Redirect and Chord's performances remain the same on the 5 link topologies. On the other hand, CAN Multidim performs differently, because part of the coordinates is influenced by the LCSs. In all cases, CAN Multi-sp has the shortest average PRD due to the ND-based partition. CAN Multi-dim stands as the second winner under most datasets, because a part of each node's coordinate is from its network coordinate. Chord Redirect occupies the third place. As the number of crawlers increases, the average PRD of all methods tends to decrease. However, the CAN-based methods changes slower than Chord Redirect, and are caught up by Chord Redirect when the number of crawlers reaches 140 . We recognize that the low decrease rate is cause by the inconsistency of CAN's space partition algorithm. Figure 12 shows a typical case of such inconsistency with 3 peers joining a 2-d coordinate space. The third Peer C's ID (coordinate) falls into the zone owned by Peer B. According to [17], Peer B splits its zone in half and assigns one half to Peer C. In the right figure of Fig. 12, Peer $\mathrm{C}$ is assigned a zone in which its ID (coordinate) is not located causing in- 

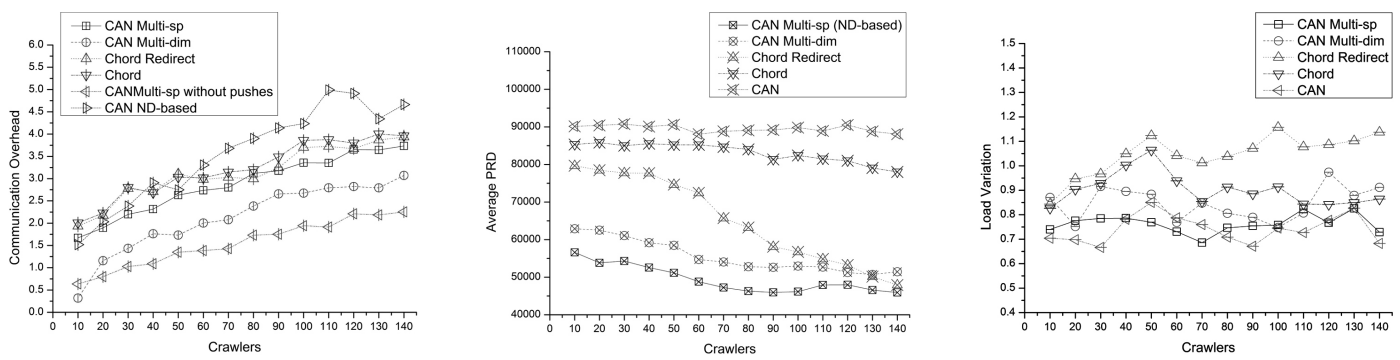

(a) real $(\mathrm{CO}, \mathrm{AP}, \mathrm{LV})$
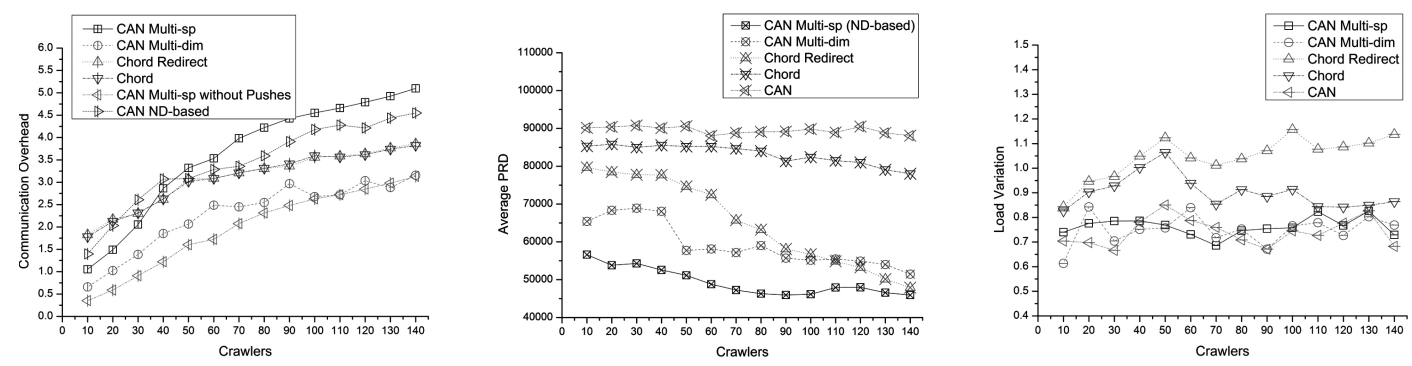

(b) BA_edge_1 (CO, AP, LV)
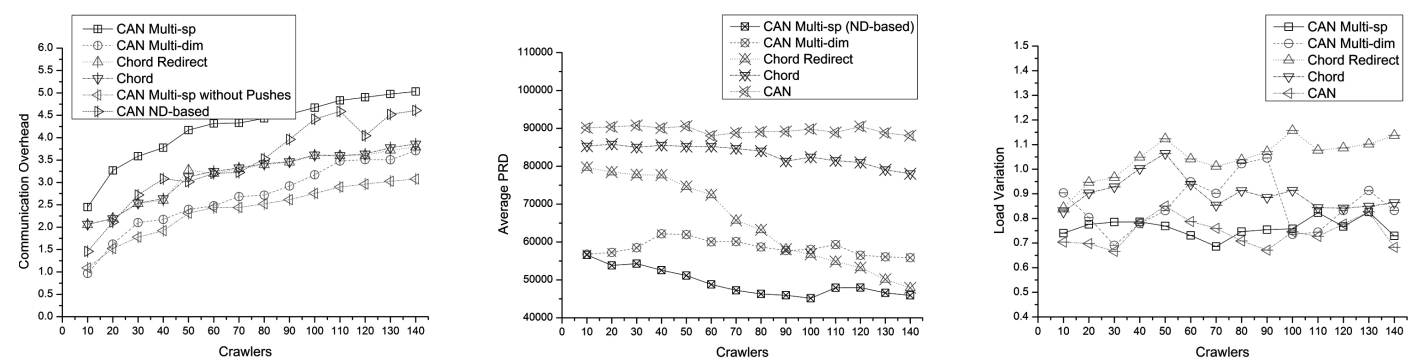

(c) BA_edge_2 (CO, AP, LV)
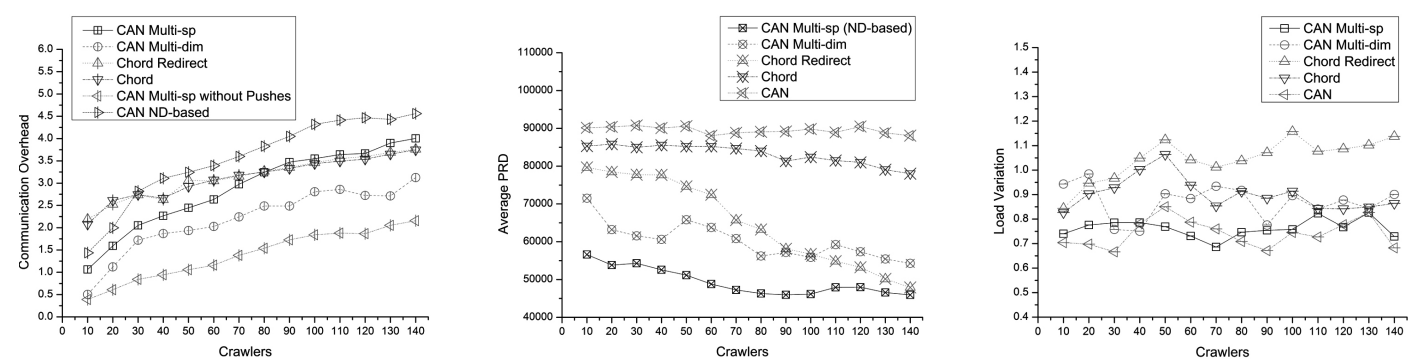

(d) Rand_edge_1 (CO, AP, LV)
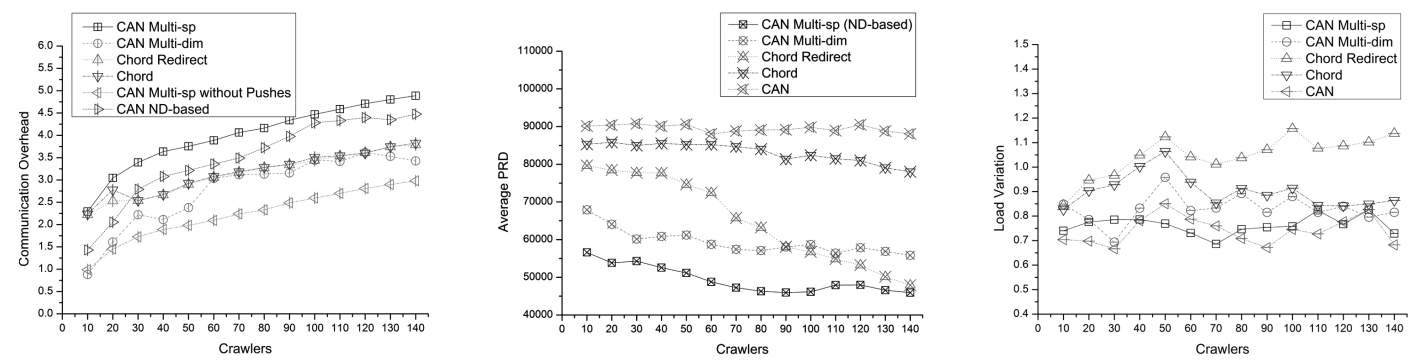

(e) Rand_edge_2 (CO, AP, LV)

Fig. 11 Comparisons on system performance. 


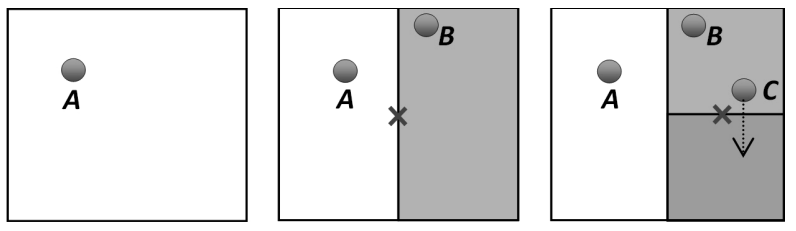

Fig. 12 The inconsistency of CAN's space partition algorithm.

consistency. We find that $70 \%$ peers are not located within their own zones when the system scale is up to 140. As a result, the crawlers can hardly be closer to the Web hosts. Such shortage can be solved by adopting a more flexible zone splitting method, e.g. split the zone through the midpoint of the two peers. And accordingly the zone recovery algorithm has to become more complicated.

In the 3rd column of Fig. 11, we assume that all crawlers have the same capacities. The load of each crawler equals to the number of Web hosts the crawler is assigned. CAN Multi-sp, CAN, Chord Redirect and Chord performs almost the same on 5 link topologies as their load distribution is not affected by the LCS. The figures are draw with no method takes load balancing into consideration. As a result, the curves are relatively volatile. To our surprise, the curves of CAN Multi-sp, CAN Multi-dim and CAN are lower than that of Chord Redirect and Chord. This indicates that CAN-based methods has more balanced load than the Chord-based ones. As CAN Multi-dim doesn't completely depend on the network coordinates, in most cases, it performs worse than CAN Multi-sp. On the other hand, adding redirect to Chord causes imbalanced load distribution. As a result, Chord Redirect performs the worst.

We conclude that, although CAN Multi-sp outperforms CAN Multi-dim in the field of average PRD, CAN Multidim shows its superiority in all the metrics against existing methods when the system scales from 10 to 100 .

\section{Conclusions and Future Works}

In this paper, a new DHT-based DWC system model is proposed. Different from the existing DHT-based models, the new model assigns each crawler and Web host a multidimensional coordinate which can be used to describe their localities on the Internet. We recognize that each Web host has 2 kinds of localities: the locality on the NC space (NCS) and the locality on the link space (LCS). First, utilizing the locality on the NCS, a ND-based partition is implemented to reduce the crawler-host network distances in a fully distributed manner. Second, utilizing the locality on the link space, we propose the concept of link-based partition to reduce the communication overhead of the system. In order to combine the benefits of both ND-based partition and link-based partition, we then propose 2 distributed multiobjective Web partition methods. Finally, all the methods we propose are compared with existing system models in the simulated experiments under different datasets and different system scales. In most cases, the new methods show their superiority.

The system model is still incomplete. First of all, we currently don't have a real world environment large enough to run a DHT-based system. There's no statistics on the realtime throughput of the system. We hope to find the solution in the near future. The second important issue is the fault tolerance and failure recovery mechanism. The failure of the system is mainly caused by the unexpected departure of the crawlers which is inevitable on the Internet. A considerable way is to replicate the Web hosts on several crawlers.

\section{Acknowledgements}

This paper was partially supported by the National Natural Science Foundation of China under Grant No.60703014; the National Grand Fundamental Research 973 Program of China under Grant No.G2005CB321806; the Specialized Research Fund for the Doctoral Program of Higher Education, SRFDP No.20070213044; the National HighTech Research and Development Plan of China under Grant Nos.2007AA01Z442; the National High-Tech Research and Development Plan of China under Grant Nos.2009AA01Z437.

\section{References}

[1] I. Foster, "Internet computing and the emerging grid," Nature Web Matters, 2000.

[2] D. Werthimer, J. Cobb, M. Lebofsky, D. Anderson, and E. Korpela, "SETI@HOME—Massively distributed computing for SETI," Comput. Sci. Eng., vol.3, pp.78-83, 2001.

[3] J. Cho and H. Garcia-Molina, "Parallel crawlers," Proc. 11th International Conference on World Wide Web, pp.124-135, New York, NY, USA, 2002.

[4] A. Heydon and M. Najork, "Mercator: A scalable, extensible Web crawler," World Wide Web, vol.2, pp.219-229, 1999.

[5] S. Brin and L. Page, "The anatomy of a large-scale hypertextual Web search engine," Computer Networks and ISDN Systems, vol.30, pp.107-117, 1998.

[6] P. Boldi, B. Codenotti, M. Santini, and S. Vigna, "Ubicrawler: A scalable fully distributed web crawler," Proc. Eighth Australian World Wide Web Conference (AUSWEB'02), 2002.

[7] D. Karger, E. Lehman, T. Leighton, R. Panigrahy, M. Levine, and D. Lewin, "Consistent hashing and random trees: distributed caching protocols for relieving hot spots on the World Wide Web," Proc. ACM Symposium on Theory of Computing, pp.654-663, New York, NY, USA, 1997.

[8] O. Papapetrou and G. Samaras, "IPMicra: Toward a distributed and adaptable location aware web crawler," Proc. ADBIS'04, Budapest, Hungary, 2004.

[9] O. Papapetrou and G. Samaras, "IPMicra: An IP-address based location aware distributed Web crawler," 5th International Conference on Internet Computing (IC 2004), pp.694-699, Las Vegas, USA, 2004.

[10] B.B. Cambazoglu, E. Karaca, T. Kucukyilmaz, A. Turk, and C. Aykanat, "Architecture of a grid-enabled Web search engine," Inf. Process. Manage., vol.43, pp.609-623, 2007.

[11] A. Singh, M. Srivatsa, L. Liu, and T. Miller, "Apoidea: A decentralized peer-to-peer architecture for crawling the world wide Web," Proc. SIGIR'03 Workshop on Distributed Information Retrieval, pp.126-142, 2003.

[12] X. Xu, W. Zhang, H. Zhang, B. Fang, and X. Liu, "A forwardingbased task scheduling algorithm for distributed Web crawling over 
DHTs,” Proc. ICPADS'09 Workshop iVCE'09, Shenzhen, China, 2001.

[13] L. Fei, M. Fan-Yuan, Y. Yun-Ming, L. Ming-Lu, and Y. Jia-Di, "IglooG: A distributed Web crawler based on grid service," Lect. Notes Comput. Sci., vol.3399, pp.207-216, 2005.

[14] "YaCy Distributed Web Search," http://yacy.net/

[15] “Majesti-12: Distributed Web Search," http://www.majestic12.co.uk/

[16] "FAROO Real Time Search," http://www.faroo.com/

[17] S. Ratnasamy, P. Francis, M. Handley, R. Karp, and S. Shenker, "A scalable content addressable network," Proc. ACM SIGCOMM'01, pp.161-172, San Diego, California, USA, 2001.

[18] S. Ratnasamy, M. Handley, R. Karp, and S. Shenker, "Topologicallyaware overlay construction and server selection," Proc. INFOCOM'02, 2002.

[19] K. Doi, S. Tagashira, and S. Fujita, "Proximity-aware content addressable network based on Vivaldi network coordinate system," Proc. Fifth International Workshop on Databases, Information Systems and Peer-to-Peer Computing, Vienna, 2007.

[20] H.Z.T.S. Eugene Ng, "Towards global network positioning," Proc. SIGCOMM Internet Measurement Workshop, San Francisco, CA, 2001.

[21] M.C.A.R. Manuel Costa, "PIC: Practical Internet coordinates for distance estimation," Proc. International Conference on Distributed Systems, Tokyo, Japan, 2004.

[22] J.C.S.W. Marcelo Pias, "Lighthouses for scalable distributed location," Proc. Second International Workshop on Peer-to-Peer Systems (IPTPS '03), 2003.

[23] F. Dabek, R. Cox, F. Kaashoek, and R. Morris, "Vivaldi: A decentralized network coordinate system," Proc. ACM SIGCOMM'04, pp.15-26, 2004.

[24] J. Exposto, J. Macedo, A. Pina, A. Alces, and J. Rufino, "Multiobjective Web partitioning for efficient distributed Web crawling," Proc. 2005 Workshop on Geographic Information Retrieval, Bremen, Germany, 2005.

[25] “SogouT-link," http://www.sogou.com/labs/dl/t-link.html

[26] “P2PSim-Kingdata," http://pdos.csail.mit.edu/p2psim/kingdata/

[27] "Network Coordinate Research at Harvard," http://www.eecs.harvard.edu/ $/$ syrah/nc/

[28] R.M.D.K. Ion Stoica, "Chord: A scalable peer-to-peer lookup service for Internet applications," Proc. SIGCOMM 2001, pp.149-160, San Diego, California, 2001.

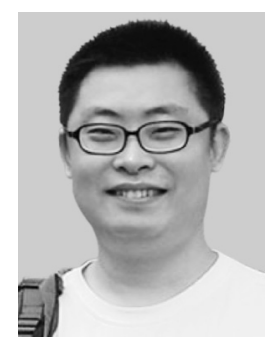

Xiao Xu received his B.S. and M.S. degrees in computer science from Harbin Institute of Technology (HIT) in 2005 and 2007. $\mathrm{He}$ is now working towards his Ph.D. degree in computer science at Harbin Institute of Technology. His research interests include computer network, distributed computing.

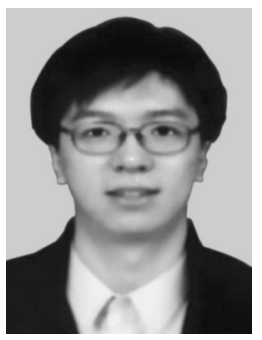

Weizhe Zhang received his B.S., M.S. and $\mathrm{Ph} . \mathrm{D}$. degrees in computer science from Harbin Institute of Technology (HIT) in 1999, 2001 and 2006 respectively. He has been working in HIT from 2002 and been an Associate Professor at computer science of HIT since 2007. His research interests include computer network, theory of computation and parallel computing.

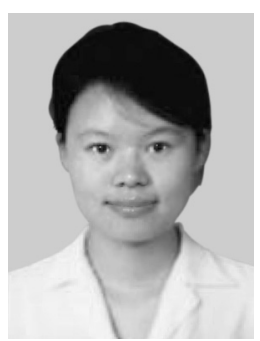

Hongli Zhang received her Ph.D. degree in computer science in 1999. She is now a Professor of school of computer science and technology of Harbin Institute of Technology (HIT). She is a member of China Computer Federation. Her research interests include network security and grid computing.

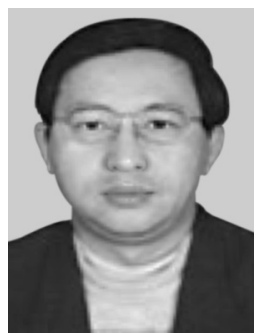

Binxing Fang received his Ph.D. in computer science in 1989. He is a Professor of school of computer science and technology of Harbin Institute of Technology (HIT). He has been a Academician of Chinese Academy of Engineering since 2005. His research interests include network security and grid computing. 